Supporting information for

\title{
Hydrogen and Deuterium Atoms in Octasilsesquioxanes: \\ Experimental and Computational Studies
}

\author{
Michael Päch, Roderick M. Macrae ${ }^{1, *}$, Ian Carmichael
}

Radiation Laboratory, University of Notre Dame. Notre Dame, Indiana 46556, USA

rmacrae@marian.edu

\section{RECEIVED DATE}

H/D detrapping from siloxane cages

1. Present address: Department of Natural and Behavioral Sciences, Marian College, Indianapolis, Indiana 46222, USA. 


\section{Data}

Cartesian coordinates (in $\AA$ ) and total energies (in hartrees) for all of the structures described in the paper.

\section{1. $\mathrm{Si}_{8} \mathrm{O}_{12} \mathrm{H}_{8}\left(\mathrm{HT}_{8}\right)$}

(a) Empty cage - optimized minimum energy geometry, B3LYP/3-21+G*

Total energy $=-3208.77210366$ hartree

$\begin{array}{llll}\mathrm{Si} & 1.600432 & 1.600432 & 1.600432 \\ \mathrm{Si} & -1.600432 & 1.600432 & 1.600432 \\ \mathrm{Si} & 1.600432 & 1.600432 & -1.600432 \\ \mathrm{Si} & 1.600432 & -1.600432 & 1.600432 \\ \mathrm{Si} & -1.600432 & 1.600432 & -1.600432 \\ \mathrm{Si} & -1.600432 & -1.600432 & 1.600432 \\ \mathrm{Si} & 1.600432 & -1.600432 & -1.600432 \\ \mathrm{Si} & -1.600432 & -1.600432 & -1.600432 \\ \mathrm{O} & 0.000000 & 1.884799 & 1.884799 \\ \mathrm{O} & 0.000000 & 1.884799 & -1.884799 \\ \mathrm{O} & 0.000000 & -1.884799 & 1.884799 \\ \mathrm{O} & 0.000000 & -1.884799 & -1.884799 \\ \mathrm{O} & 1.884799 & 1.884799 & 0.000000 \\ \mathrm{O} & -1.884799 & 1.884799 & 0.000000 \\ \mathrm{O} & 1.884799 & -1.884799 & 0.000000 \\ \mathrm{O} & -1.884799 & -1.884799 & 0.000000 \\ \mathrm{O} & 1.884799 & 0.000000 & 1.884799 \\ \mathrm{O} & -1.884799 & 0.000000 & 1.884799 \\ \mathrm{O} & 1.884799 & 0.000000 & -1.884799 \\ \mathrm{O} & -1.884799 & 0.000000 & -1.884799 \\ \mathrm{H} & 2.445524 & 2.445524 & 2.445524 \\ \mathrm{H} & -2.445524 & 2.445524 & 2.445524 \\ \mathrm{H} & 2.445524 & 2.445524 & -2.445524 \\ \mathrm{H} & 2.445524 & -2.445524 & 2.445524 \\ \mathrm{H} & -2.445524 & 2.445524 & -2.445524 \\ \mathrm{H} & -2.445524 & -2.445524 & 2.445524 \\ \mathrm{H} & 2.445524 & -2.445524 & -2.445524 \\ \mathrm{H} & -2.445524 & -2.445524 & -2.445524\end{array}$

(b) Empty cage - optimized minimum energy geometry, B3LYP/6-31+G*

Total energy $=-3224.70307649$ hartree 


$\begin{array}{llll}\mathrm{Si} & 1.583181 & 1.583181 & 1.583181 \\ \mathrm{Si} & -1.583181 & 1.583181 & 1.583181 \\ \mathrm{Si} & 1.583181 & 1.583181 & -1.583181 \\ \mathrm{Si} & 1.583181 & -1.583181 & 1.583181 \\ \mathrm{Si} & -1.583181 & 1.583181 & -1.583181 \\ \mathrm{Si} & -1.583181 & -1.583181 & 1.583181 \\ \mathrm{Si} & 1.583181 & -1.583181 & -1.583181 \\ \mathrm{Si} & -1.583181 & -1.583181 & -1.583181 \\ \mathrm{O} & 0.000000 & 1.900362 & 1.900362 \\ \mathrm{O} & 0.000000 & 1.900362 & -1.900362 \\ \mathrm{O} & 0.000000 & -1.900362 & 1.900362 \\ \mathrm{O} & 0.000000 & -1.900362 & -1.900362 \\ \mathrm{O} & 1.900362 & 1.900362 & 0.000000 \\ \mathrm{O} & -1.900362 & 1.900362 & 0.000000 \\ \mathrm{O} & 1.900362 & -1.900362 & 0.000000 \\ \mathrm{O} & -1.900362 & -1.900362 & 0.000000 \\ \mathrm{O} & 1.900362 & 0.000000 & 1.900362 \\ \mathrm{O} & -1.900362 & 0.000000 & 1.900362 \\ \mathrm{O} & 1.900362 & 0.000000 & -1.900362 \\ \mathrm{O} & -1.900362 & 0.000000 & -1.900362 \\ \mathrm{H} & 2.428326 & 2.428326 & 2.428326 \\ \mathrm{H} & -2.428326 & 2.428326 & 2.428326 \\ \mathrm{H} & 2.428326 & 2.428326 & -2.428326 \\ \mathrm{H} & 2.428326 & -2.428326 & 2.428326 \\ \mathrm{H} & -2.428326 & 2.428326 & -2.428326 \\ \mathrm{H} & -2.428326 & -2.428326 & 2.428326 \\ \mathrm{H} & 2.428326 & -2.428326 & -2.428326 \\ \mathrm{H} & -2.428326 & -2.428326 & -2.428326\end{array}$

(c) Filled cage - optimized minimum energy geometry, UB3LYP/3-21+G*

Total energy $=-3209.25747527$ hartree

$\begin{array}{llll}\mathrm{Si} & 1.601305 & 1.601305 & 1.601305 \\ \mathrm{Si} & -1.601305 & 1.601305 & 1.601305 \\ \mathrm{Si} & 1.601305 & 1.601305 & -1.601305 \\ \mathrm{Si} & 1.601305 & -1.601305 & 1.601305 \\ \mathrm{Si} & -1.601305 & 1.601305 & -1.601305 \\ \mathrm{Si} & -1.601305 & -1.601305 & 1.601305 \\ \mathrm{Si} & 1.601305 & -1.601305 & -1.601305 \\ \mathrm{Si} & -1.601305 & -1.601305 & -1.601305 \\ \mathrm{O} & 0.000000 & 1.891137 & 1.891137 \\ \mathrm{O} & 0.000000 & 1.891137 & -1.891137 \\ \mathrm{O} & 0.000000 & -1.891137 & 1.891137 \\ \mathrm{O} & 0.000000 & -1.891137 & -1.891137 \\ \mathrm{O} & 1.891137 & 1.891137 & 0.000000\end{array}$




$\begin{array}{llll}\mathrm{O} & -1.891137 & 1.891137 & 0.000000 \\ \mathrm{O} & 1.891137 & -1.891137 & 0.000000 \\ \mathrm{O} & -1.891137 & -1.891137 & 0.000000 \\ \mathrm{O} & 1.891137 & 0.000000 & 1.891137 \\ \mathrm{O} & -1.891137 & 0.000000 & 1.891137 \\ \mathrm{O} & 1.891137 & 0.000000 & -1.891137 \\ \mathrm{O} & -1.891137 & 0.000000 & -1.891137 \\ \mathrm{H} & 2.446290 & 2.446290 & 2.446290 \\ \mathrm{H} & -2.446290 & 2.446290 & 2.446290 \\ \mathrm{H} & 2.446290 & 2.446290 & -2.446290 \\ \mathrm{H} & 2.446290 & -2.446290 & 2.446290 \\ \mathrm{H} & -2.446290 & 2.446290 & -2.446290 \\ \mathrm{H} & -2.446290 & -2.446290 & 2.446290 \\ \mathrm{H} & 2.446290 & -2.446290 & -2.446290 \\ \mathrm{H} & -2.446290 & -2.446290 & -2.446290 \\ \mathrm{H} & 0.000000 & 0.000000 & 0.000000\end{array}$

(d) Filled cage - optimized minimum energy geometry, UB3LYP/6-31+G*

Total energy $=-3225.19075087$ hartree

$\begin{array}{llll}\mathrm{Si} & 1.583990 & 1.583990 & 1.583990 \\ \mathrm{Si} & -1.583990 & 1.583990 & 1.583990 \\ \mathrm{Si} & 1.583990 & 1.583990 & -1.583990 \\ \mathrm{Si} & 1.583990 & -1.583990 & 1.583990 \\ \mathrm{Si} & -1.583990 & 1.583990 & -1.583990 \\ \mathrm{Si} & -1.583990 & -1.583990 & 1.583990 \\ \mathrm{Si} & 1.583990 & -1.583990 & -1.583990 \\ \mathrm{Si} & -1.583990 & -1.583990 & -1.583990 \\ \mathrm{O} & 0.000000 & 1.905348 & 1.905348 \\ \mathrm{O} & 0.000000 & 1.905348 & -1.905348 \\ \mathrm{O} & 0.000000 & -1.905348 & 1.905348 \\ \mathrm{O} & 0.000000 & -1.905348 & -1.905348 \\ \mathrm{O} & 1.905348 & 1.905348 & 0.000000 \\ \mathrm{O} & -1.905348 & 1.905348 & 0.000000 \\ \mathrm{O} & 1.905348 & -1.905348 & 0.000000 \\ \mathrm{O} & -1.905348 & -1.905348 & 0.000000 \\ \mathrm{O} & 1.905348 & 0.000000 & 1.905348 \\ \mathrm{O} & -1.905348 & 0.000000 & 1.905348 \\ \mathrm{O} & 1.905348 & 0.000000 & -1.905348 \\ \mathrm{O} & -1.905348 & 0.000000 & -1.905348 \\ \mathrm{H} & 2.429028 & 2.429028 & 2.429028 \\ \mathrm{H} & -2.429028 & 2.429028 & 2.429028 \\ \mathrm{H} & 2.429028 & 2.429028 & -2.429028 \\ \mathrm{H} & 2.429028 & -2.429028 & 2.429028 \\ \mathrm{H} & -2.429028 & 2.429028 & -2.429028\end{array}$




$\begin{array}{llll}\mathrm{H} & -2.429028 & -2.429028 & 2.429028 \\ \mathrm{H} & 2.429028 & -2.429028 & -2.429028 \\ \mathrm{H} & -2.429028 & -2.429028 & -2.429028 \\ \mathrm{H} & 0.000000 & 0.000000 & 0.000000\end{array}$

(e) Filled cage - optimized minimum energy geometry, UHF/6-31+G*

Total energy $=-3216.20446796$ hartree

$\begin{array}{llll}\mathrm{H} & 0.000000 & 0.000000 & 0.000000 \\ \mathrm{Si} & 1.570140 & 1.570140 & 1.570140 \\ \mathrm{Si} & -1.57014 & 1.57014 & 1.57014 \\ \mathrm{Si} & 1.57014 & 1.57014 & -1.57014 \\ \mathrm{Si} & 1.57014 & -1.57014 & 1.57014 \\ \mathrm{Si} & -1.57014 & 1.57014 & -1.57014 \\ \mathrm{Si} & -1.57014 & -1.57014 & 1.57014 \\ \mathrm{Si} & 1.57014 & -1.57014 & -1.57014 \\ \mathrm{Si} & -1.57014 & -1.57014 & -1.57014 \\ \mathrm{O} & 0.000000 & 1.875647 & 1.875647 \\ \mathrm{O} & 0.000000 & 1.875647 & -1.875647 \\ \mathrm{O} & 0.000000 & -1.875647 & 1.875647 \\ \mathrm{O} & 0.000000 & -1.875647 & -1.875647 \\ \mathrm{O} & 1.875647 & 1.875647 & 0.000000 \\ \mathrm{O} & -1.875647 & 1.875647 & 0.000000 \\ \mathrm{O} & 1.875647 & -1.875647 & 0.000000 \\ \mathrm{O} & -1.875647 & -1.875647 & 0.000000 \\ \mathrm{O} & 1.875647 & 0.000000 & 1.875647 \\ \mathrm{O} & -1.875647 & 0.000000 & 1.875647 \\ \mathrm{O} & 1.875647 & 0.000000 & -1.875647 \\ \mathrm{O} & -1.875647 & 0.000000 & -1.875647 \\ \mathrm{H} & 2.408847 & 2.408847 & 2.408847 \\ \mathrm{H} & -2.408847 & 2.408847 & 2.408847 \\ \mathrm{H} & 2.408847 & 2.408847 & -2.408847 \\ \mathrm{H} & 2.408847 & -2.408847 & 2.408847 \\ \mathrm{H} & -2.408847 & 2.408847 & -2.408847 \\ \mathrm{H} & -2.408847 & -2.408847 & 2.408847 \\ \mathrm{H} & 2.408847 & -2.408847 & -2.408847 \\ \mathrm{H} & -2.408847 & -2.408847 & -2.408847\end{array}$

(f) Filled cage - optimized minimum energy geometry, UB-PW91/6-31+G*

Total energy $=-3224.92520520$ hartree

$\begin{array}{llll}\mathrm{H} & 0.000000 & 0.000000 & 0.000000 \\ \mathrm{Si} & 1.590114 & 1.590114 & 1.590114 \\ \mathrm{Si} & -1.590114 & 1.590114 & 1.590114\end{array}$




$\begin{array}{llll}\mathrm{Si} & 1.590114 & 1.590114 & -1.590114 \\ \mathrm{Si} & 1.590114 & -1.590114 & 1.590114 \\ \mathrm{Si} & -1.590114 & 1.590114 & -1.590114 \\ \mathrm{Si} & -1.590114 & -1.590114 & 1.590114 \\ \mathrm{Si} & 1.590114 & -1.590114 & -1.590114 \\ \mathrm{Si} & -1.590114 & -1.590114 & -1.590114 \\ \mathrm{O} & 0.000000 & 1.925969 & 1.925969 \\ \mathrm{O} & 0.000000 & 1.925969 & -1.925969 \\ \mathrm{O} & 0.000000 & -1.925969 & 1.925969 \\ \mathrm{O} & 0.000000 & -1.925969 & -1.925969 \\ \mathrm{O} & 1.925969 & 1.925969 & 0.000000 \\ \mathrm{O} & -1.925969 & 1.925969 & 0.000000 \\ \mathrm{O} & 1.925969 & -1.925969 & 0.000000 \\ \mathrm{O} & -1.925969 & -1.925969 & 0.000000 \\ \mathrm{O} & 1.925969 & 0.000000 & 1.925969 \\ \mathrm{O} & -1.925969 & 0.000000 & 1.925969 \\ \mathrm{O} & 1.925969 & 0.000000 & -1.925969 \\ \mathrm{O} & -1.925969 & 0.000000 & -1.925969 \\ \mathrm{H} & 2.441572 & 2.441572 & 2.441572 \\ \mathrm{H} & -2.441572 & 2.441572 & 2.441572 \\ \mathrm{H} & 2.441572 & 2.441572 & -2.441572 \\ \mathrm{H} & 2.441572 & -2.441572 & 2.441572 \\ \mathrm{H} & -2.441572 & 2.441572 & -2.441572 \\ \mathrm{H} & -2.441572 & -2.441572 & 2.441572 \\ \mathrm{H} & 2.441572 & -2.441572 & -2.441572 \\ \mathrm{H} & -2.441572 & -2.441572 & -2.441572\end{array}$

(g) H atom cage exit transition state, UB3LYP/3-21+G*

Total energy $=-3209.21653101$ hartree

$\begin{array}{llll}\mathrm{Si} & 0.000000 & 2.253254 & 1.613177 \\ \mathrm{Si} & 2.253254 & 0.000000 & 1.613177 \\ \mathrm{Si} & -2.253254 & 0.000000 & 1.613177 \\ \mathrm{Si} & 0.000000 & -2.253254 & 1.613177 \\ \mathrm{Si} & 0.000000 & 2.242833 & -1.631589 \\ \mathrm{Si} & 2.242833 & 0.000000 & -1.631589 \\ \mathrm{Si} & -2.242833 & 0.000000 & -1.631589 \\ \mathrm{Si} & 0.000000 & -2.242833 & -1.631589 \\ \mathrm{O} & 0.000000 & 2.533158 & -0.008842 \\ \mathrm{O} & 2.533158 & 0.000000 & -0.008842 \\ \mathrm{O} & -2.533158 & 0.000000 & -0.008842 \\ \mathrm{O} & 0.000000 & -2.533158 & -0.008842 \\ \mathrm{O} & 1.405810 & 1.405810 & 1.953091 \\ \mathrm{O} & -1.405810 & -1.405810 & 1.953091\end{array}$




$\begin{array}{llll}\mathrm{O} & -1.405810 & 1.405810 & 1.953091 \\ \mathrm{O} & 1.405810 & -1.405810 & 1.953091 \\ \mathrm{O} & 1.335382 & 1.335382 & -1.972996 \\ \mathrm{O} & -1.335382 & 1.335382 & -1.972996 \\ \mathrm{O} & 1.335382 & -1.335382 & -1.972996 \\ \mathrm{O} & -1.335382 & -1.335382 & -1.972996 \\ \mathrm{H} & 0.000000 & 3.494606 & 2.387661 \\ \mathrm{H} & 3.494606 & 0.000000 & 2.387661 \\ \mathrm{H} & -3.494606 & 0.000000 & 2.387661 \\ \mathrm{H} & 0.000000 & -3.494606 & 2.387661 \\ \mathrm{H} & 3.486352 & 0.000000 & -2.403704 \\ \mathrm{H} & -3.486352 & 0.000000 & -2.403704 \\ \mathrm{H} & 0.000000 & 3.486352 & -2.403704 \\ \mathrm{H} & 0.000000 & -3.486352 & -2.403704 \\ \mathrm{H} & 0.000000 & 0.000000 & 2.015128\end{array}$

(h) $\mathrm{H}$ atom cage exit transition state, UB3LYP/6-31+G*

Total energy $=-3225.15167527$ hartree

$\begin{array}{llll}\mathrm{Si} & 0.000000 & 2.233037 & 1.595188 \\ \mathrm{Si} & 2.233037 & 0.000000 & 1.595188 \\ \mathrm{Si} & -2.233037 & 0.000000 & 1.595188 \\ \mathrm{Si} & 0.000000 & -2.233037 & 1.595188 \\ \mathrm{Si} & 0.000000 & 2.222028 & -1.611372 \\ \mathrm{Si} & 2.222028 & 0.000000 & -1.611372 \\ \mathrm{Si} & -2.222028 & 0.000000 & -1.611372 \\ \mathrm{Si} & 0.000000 & -2.222028 & -1.611372 \\ \mathrm{O} & 0.000000 & 2.582921 & -0.008346 \\ \mathrm{O} & 2.582921 & 0.000000 & -0.008346 \\ \mathrm{O} & -2.582921 & 0.000000 & -0.008346 \\ \mathrm{O} & 0.000000 & -2.582921 & -0.008346 \\ \mathrm{O} & 1.411986 & 1.411986 & 1.949223 \\ \mathrm{O} & -1.411986 & -1.411986 & 1.949223 \\ \mathrm{O} & -1.411986 & 1.411986 & 1.949223 \\ \mathrm{O} & 1.411986 & -1.411986 & 1.949223 \\ \mathrm{O} & 1.346024 & 1.346024 & -1.974069 \\ \mathrm{O} & -1.346024 & 1.346024 & -1.974069 \\ \mathrm{O} & 1.346024 & -1.346024 & -1.974069 \\ \mathrm{O} & -1.346024 & -1.346024 & -1.974069 \\ \mathrm{H} & 0.000000 & 3.463627 & 2.387004 \\ \mathrm{H} & 3.463627 & 0.000000 & 2.387004 \\ \mathrm{H} & -3.463627 & 0.000000 & 2.387004 \\ \mathrm{H} & 0.000000 & -3.463627 & 2.387004 \\ \mathrm{H} & 3.456964 & 0.000000 & -2.397240 \\ \mathrm{H} & -3.456964 & 0.000000 & -2.397240\end{array}$




$\begin{array}{llll}\mathrm{H} & 0.000000 & 3.456964 & -2.397240 \\ \mathrm{H} & 0.000000 & -3.456964 & -2.397240 \\ \mathrm{H} & 0.000000 & 0.000000 & 2.009412\end{array}$

\section{2. $\mathrm{Si}_{8} \mathrm{O12}\left(\mathrm{CH}_{3}\right) 8\left(\mathrm{MeT}_{8}\right)$}

(a) Empty cage - optimized minimum energy geometry, B3LYP/6-31+G*

Total energy $=-3539.37321526$ hartree

$\begin{array}{lrrr}\mathrm{Si} & 1.589553 & 1.589553 & 1.589553 \\ \mathrm{Si} & -1.589553 & 1.589553 & 1.589553 \\ \mathrm{Si} & 1.589553 & 1.589553 & -1.589553 \\ \mathrm{Si} & 1.589553 & -1.589553 & 1.589553 \\ \mathrm{Si} & -1.589553 & 1.589553 & -1.589553 \\ \mathrm{Si} & -1.589553 & -1.589553 & 1.589553 \\ \mathrm{Si} & 1.589553 & -1.589553 & -1.589553 \\ \mathrm{Si} & -1.589553 & -1.589553 & -1.589553 \\ \mathrm{O} & 0.000000 & 1.899825 & 1.899825 \\ \mathrm{O} & 0.000000 & 1.899825 & -1.899825 \\ \mathrm{O} & 0.000000 & -1.899825 & 1.899825 \\ \mathrm{O} & 0.000000 & -1.899825 & -1.899825 \\ \mathrm{O} & 1.899825 & 1.899825 & 0.000000 \\ \mathrm{O} & -1.899825 & 1.899825 & 0.000000 \\ \mathrm{O} & 1.899825 & -1.899825 & 0.000000 \\ \mathrm{O} & -1.899825 & -1.899825 & 0.000000 \\ \mathrm{O} & 1.899825 & 0.000000 & 1.899825 \\ \mathrm{O} & -1.899825 & 0.000000 & 1.899825 \\ \mathrm{O} & 1.899825 & 0.000000 & -1.899825 \\ \mathrm{O} & -1.899825 & 0.000000 & -1.899825 \\ \mathrm{C} & 2.661407 & 2.661407 & 2.661407 \\ \mathrm{C} & -2.661407 & 2.661407 & 2.661407 \\ \mathrm{C} & 2.661407 & 2.661407 & -2.661407 \\ \mathrm{C} & 2.661407 & -2.661407 & 2.661407 \\ \mathrm{C} & -2.661407 & 2.661407 & -2.661407 \\ \mathrm{C} & -2.661407 & -2.661407 & 2.661407 \\ \mathrm{C} & 2.661407 & -2.661407 & -2.661407 \\ \mathrm{C} & -2.661407 & -2.661407 & -2.661407 \\ \mathrm{H} & 3.722349 & 2.467271 & 2.467271 \\ \mathrm{H} & 2.467271 & 3.722349 & 2.467271 \\ \mathrm{H} & 2.467271 & 2.467271 & 3.722349 \\ \mathrm{H} & -3.722349 & 2.467271 & 2.467271 \\ \mathrm{H} & -2.467271 & 3.722349 & 2.467271 \\ \mathrm{H} & -2.467271 & 2.467271 & 3.722349 \\ \mathrm{H} & 3.722349 & 2.467271 & -2.467271 \\ \mathrm{H} & 2.467271 & 3.722349 & -2.467271\end{array}$




$\begin{array}{lrrr}\mathrm{H} & 2.467271 & 2.467271 & -3.722349 \\ \mathrm{H} & 3.722349 & -2.467271 & 2.467271 \\ \mathrm{H} & 2.467271 & -3.722349 & 2.467271 \\ \mathrm{H} & 2.467271 & -2.467271 & 3.722349 \\ \mathrm{H} & -3.722349 & 2.467271 & -2.467271 \\ \mathrm{H} & -2.467271 & 3.722349 & -2.467271 \\ \mathrm{H} & -2.467271 & 2.467271 & -3.722349 \\ \mathrm{H} & -3.722349 & -2.467271 & 2.467271 \\ \mathrm{H} & -2.467271 & -3.722349 & 2.467271 \\ \mathrm{H} & -2.467271 & -2.467271 & 3.722349 \\ \mathrm{H} & 3.722349 & -2.467271 & -2.467271 \\ \mathrm{H} & 2.467271 & -3.722349 & -2.467271 \\ \mathrm{H} & 2.467271 & -2.467271 & -3.722349 \\ \mathrm{H} & -3.722349 & -2.467271 & -2.467271 \\ \mathrm{H} & -2.467271 & -3.722349 & -2.467271 \\ \mathrm{H} & -2.467271 & -2.467271 & -3.722349\end{array}$

(b) Empty cage - optimized minimum energy geometry, B3LYP/3-21+G*

Total energy $=-3521.82177666$ hartree

$\begin{array}{llll}\mathrm{Si} & 1.603417 & 1.603417 & 1.603417 \\ \mathrm{Si} & -1.603417 & 1.603417 & 1.603417 \\ \mathrm{Si} & 1.603417 & 1.603417 & -1.603417 \\ \mathrm{Si} & 1.603417 & -1.603417 & 1.603417 \\ \mathrm{Si} & -1.603417 & 1.603417 & -1.603417 \\ \mathrm{Si} & -1.603417 & -1.603417 & 1.603417 \\ \mathrm{Si} & 1.603417 & -1.603417 & -1.603417 \\ \mathrm{Si} & -1.603417 & -1.603417 & -1.603417 \\ \mathrm{O} & 0.000000 & 1.884157 & 1.884157 \\ \mathrm{O} & 0.000000 & 1.884157 & -1.884157 \\ \mathrm{O} & 0.000000 & -1.884157 & 1.884157 \\ \mathrm{O} & 0.000000 & -1.884157 & -1.884157 \\ \mathrm{O} & 1.884157 & 1.884157 & 0.000000 \\ \mathrm{O} & -1.884157 & 1.884157 & 0.000000 \\ \mathrm{O} & 1.884157 & -1.884157 & 0.000000 \\ \mathrm{O} & -1.884157 & -1.884157 & 0.000000 \\ \mathrm{O} & 1.884157 & 0.000000 & 1.884157 \\ \mathrm{O} & -1.884157 & 0.000000 & 1.884157 \\ \mathrm{O} & 1.884157 & 0.000000 & -1.884157 \\ \mathrm{O} & -1.884157 & 0.000000 & -1.884157 \\ \mathrm{C} & 2.670872 & 2.670872 & 2.670872 \\ \mathrm{C} & -2.670872 & 2.670872 & 2.670872 \\ \mathrm{C} & 2.670872 & 2.670872 & -2.670872 \\ \mathrm{C} & 2.670872 & -2.670872 & 2.670872 \\ \mathrm{C} & -2.670872 & 2.670872 & -2.670872\end{array}$




$\begin{array}{llll}\mathrm{C} & -2.670872 & -2.670872 & 2.670872 \\ \mathrm{C} & 2.670872 & -2.670872 & -2.670872 \\ \mathrm{C} & -2.670872 & -2.670872 & -2.670872 \\ \mathrm{H} & 3.734205 & 2.476022 & 2.476022 \\ \mathrm{H} & 2.476022 & 3.734205 & 2.476022 \\ \mathrm{H} & 2.476022 & 2.476022 & 3.734205 \\ \mathrm{H} & -3.734205 & 2.476022 & 2.476022 \\ \mathrm{H} & -2.476022 & 3.734205 & 2.476022 \\ \mathrm{H} & -2.476022 & 2.476022 & 3.734205 \\ \mathrm{H} & 3.734205 & 2.476022 & -2.476022 \\ \mathrm{H} & 2.476022 & 3.734205 & -2.476022 \\ \mathrm{H} & 2.476022 & 2.476022 & -3.734205 \\ \mathrm{H} & 3.734205 & -2.476022 & 2.476022 \\ \mathrm{H} & 2.476022 & -3.734205 & 2.476022 \\ \mathrm{H} & 2.476022 & -2.476022 & 3.734205 \\ \mathrm{H} & -3.734205 & 2.476022 & -2.476022 \\ \mathrm{H} & -2.476022 & 3.734205 & -2.476022 \\ \mathrm{H} & -2.476022 & 2.476022 & -3.734205 \\ \mathrm{H} & -3.734205 & -2.476022 & 2.476022 \\ \mathrm{H} & -2.476022 & -3.734205 & 2.476022 \\ \mathrm{H} & -2.476022 & -2.476022 & 3.734205 \\ \mathrm{H} & 3.734205 & -2.476022 & -2.476022 \\ \mathrm{H} & 2.476022 & -3.734205 & -2.476022 \\ \mathrm{H} & 2.476022 & -2.476022 & -3.734205 \\ \mathrm{H} & -3.734205 & -2.476022 & -2.476022 \\ \mathrm{H} & -2.476022 & -3.734205 & -2.476022 \\ \mathrm{H} & -2.476022 & -2.476022 & -3.734205 \\ & & & \end{array}$

(c) Empty cage - eclipsed methyl rotamer, B3LYP/3-21+G*

Total energy $=-3521.81268270$ hartree

$\begin{array}{llll}\mathrm{Si} & 1.604512 & 1.604512 & 1.604512 \\ \mathrm{Si} & -1.604512 & 1.604512 & 1.604512 \\ \mathrm{Si} & 1.604512 & 1.604512 & -1.604512 \\ \mathrm{Si} & 1.604512 & -1.604512 & 1.604512 \\ \mathrm{Si} & -1.604512 & 1.604512 & -1.604512 \\ \mathrm{Si} & -1.604512 & -1.604512 & 1.604512 \\ \mathrm{Si} & 1.604512 & -1.604512 & -1.604512 \\ \mathrm{Si} & -1.604512 & -1.604512 & -1.604512 \\ \mathrm{O} & 0.000000 & 1.883697 & 1.883697 \\ \mathrm{O} & 0.000000 & 1.883697 & -1.883697 \\ \mathrm{O} & 0.000000 & -1.883697 & 1.883697 \\ \mathrm{O} & 0.000000 & -1.883697 & -1.883697 \\ \mathrm{O} & 1.883697 & 1.883697 & 0.000000 \\ \mathrm{O} & -1.883697 & 1.883697 & 0.000000\end{array}$




$\begin{array}{llll}\mathrm{O} & 1.883697 & -1.883697 & 0.000000 \\ \mathrm{O} & -1.883697 & -1.883697 & 0.000000 \\ \mathrm{O} & 1.883697 & 0.000000 & 1.883697 \\ \mathrm{O} & -1.883697 & 0.000000 & 1.883697 \\ \mathrm{O} & 1.883697 & 0.000000 & -1.883697 \\ \mathrm{O} & -1.883697 & 0.000000 & -1.883697 \\ \mathrm{C} & 2.676326 & 2.676326 & 2.676326 \\ \mathrm{C} & -2.676326 & 2.676326 & 2.676326 \\ \mathrm{C} & 2.676326 & 2.676326 & -2.676326 \\ \mathrm{C} & 2.676326 & -2.676326 & 2.676326 \\ \mathrm{C} & -2.676326 & 2.676326 & -2.676326 \\ \mathrm{C} & -2.676326 & -2.676326 & 2.676326 \\ \mathrm{C} & 2.676326 & -2.676326 & -2.676326 \\ \mathrm{C} & -2.676326 & -2.676326 & -2.676326 \\ \mathrm{H} & 2.063787 & 3.320742 & 3.320742 \\ \mathrm{H} & 3.320742 & 2.063787 & 3.320742 \\ \mathrm{H} & 3.320742 & 3.320742 & 2.063787 \\ \mathrm{H} & -2.063787 & 3.320742 & 3.320742 \\ \mathrm{H} & -3.320742 & 2.063787 & 3.320742 \\ \mathrm{H} & -3.320742 & 3.320742 & 2.063787 \\ \mathrm{H} & 2.063787 & 3.320742 & -3.320742 \\ \mathrm{H} & 3.320742 & 2.063787 & -3.320742 \\ \mathrm{H} & 3.320742 & 3.320742 & -2.063787 \\ \mathrm{H} & 2.063787 & -3.320742 & 3.320742 \\ \mathrm{H} & 3.320742 & -2.063787 & 3.320742 \\ \mathrm{H} & 3.320742 & -3.320742 & 2.063787 \\ \mathrm{H} & -2.063787 & 3.320742 & -3.320742 \\ \mathrm{H} & -3.320742 & 2.063787 & -3.320742 \\ \mathrm{H} & -3.320742 & 3.320742 & -2.063787 \\ \mathrm{H} & -2.063787 & -3.320742 & 3.320742 \\ \mathrm{H} & -3.320742 & -2.063787 & 3.320742 \\ \mathrm{H} & -3.320742 & -3.320742 & 2.063787 \\ \mathrm{H} & 2.063787 & -3.320742 & -3.320742 \\ \mathrm{H} & 3.320742 & -2.063787 & -3.320742 \\ \mathrm{H} & 3.320742 & -3.320742 & -2.063787 \\ \mathrm{H} & -2.063787 & -3.320742 & -3.320742 \\ \mathrm{H} & -3.320742 & -2.063787 & -3.320742 \\ \mathrm{H} & -3.320742 & -3.320742 & -2.063787\end{array}$

(d) Filled cage - optimized minimum energy geometry, UB3LYP/3-21+G*

Total energy $=-3522.30713023$ hartree

$\begin{array}{llll}\mathrm{Si} & -2.728100 & -0.468784 & -0.265741 \\ \mathrm{C} & -4.541513 & -0.77990 & -0.444179 \\ \mathrm{O} & -1.951989 & -0.598441 & -1.720855\end{array}$




$\begin{array}{llll}\mathrm{Si} & -0.590576 & -0.547768 & -2.659279 \\ \mathrm{C} & -0.984335 & -0.910735 & -4.428641 \\ \mathrm{O} & 0.079006 & 0.955897 & -2.492889 \\ \mathrm{Si} & 0.724862 & 2.17176 & -1.575728 \\ \mathrm{C} & 1.208348 & 3.616494 & -2.622755 \\ \mathrm{O} & -0.405611 & 2.601971 & -0.447186 \\ \mathrm{Si} & -1.412041 & 2.250299 & 0.817984 \\ \mathrm{C} & -2.34834 & 3.748072 & 1.363025 \\ \mathrm{O} & -2.437087 & 1.048848 & 0.325442 \\ \mathrm{O} & 2.031477 & 1.553258 & -0.771121 \\ \mathrm{Si} & 2.727804 & 0.468077 & 0.265516 \\ \mathrm{C} & 4.541816 & 0.778266 & 0.439974 \\ \mathrm{O} & 1.954916 & 0.599681 & 1.722154 \\ \mathrm{Si} & 0.59246 & 0.548713 & 2.658992 \\ \mathrm{C} & 0.984412 & 0.911225 & 4.428784 \\ \mathrm{O} & -0.481467 & 1.647226 & 2.045574 \\ \mathrm{O} & -0.077143 & -0.954594 & 2.490145 \\ \mathrm{Si} & -0.725696 & -2.171111 & 1.575818 \\ \mathrm{C} & -1.208075 & -3.613689 & 2.626376 \\ \mathrm{O} & -2.033228 & -1.553756 & 0.772047 \\ \mathrm{O} & 0.401632 & -2.603727 & 0.44501 \\ \mathrm{Si} & 1.411105 & -2.251489 & -0.817545 \\ \mathrm{C} & 2.347532 & -3.749496 & -1.361653 \\ \mathrm{O} & 0.484398 & -1.645689 & -2.046785 \\ \mathrm{O} & 2.435593 & -1.05049 & -0.322675 \\ \mathrm{H} & -4.726148 & -1.787514 & -0.840583 \\ \mathrm{H} & -4.994798 & -0.052722 & -1.131421 \\ \mathrm{H} & -5.047644 & -0.695237 & 0.526999 \\ \mathrm{H} & -0.074607 & -0.878712 & -5.043408 \\ \mathrm{H} & -1.693765 & -0.17313 & -4.827625 \\ \mathrm{H} & -1.432681 & -1.9082 & -4.531426 \\ \mathrm{H} & -0.334795 & -4.019616 & 3.154648 \\ \mathrm{H} & 1.635104 & 4.418308 & -2.00509 \\ \mathrm{H} & 0.336466 & 4.020423 & -3.15485 \\ \mathrm{H} & 1.95913 & 3.322113 & -3.368603 \\ \mathrm{H} & -3.008879 & 3.508335 & 2.207233 \\ \mathrm{H} & -2.966569 & 4.139789 & 0.543993 \\ \mathrm{H} & -1.658989 & 4.541685 & 1.681815 \\ \mathrm{H} & 4.997275 & 0.046933 & 1.121342 \\ \mathrm{H} & 4.727928 & 1.783493 & 0.84168 \\ \mathrm{H} & 5.044756 & 0.699288 & -0.533361 \\ \mathrm{H} & 0.073461 & 0.883988 & 5.041963 \\ \mathrm{H} & 1.436977 & 1.906825 & 4.531238 \\ \mathrm{H} & -1.640085 & -4.414869 & 2.011538 \\ \mathrm{H} & & & 3.375583 \\ \mathrm{H} & -1.954388 & -3.3165 & \\ \mathrm{H} & & \end{array}$




$\begin{array}{llll}\mathrm{H} & 3.01165 & -3.509228 & -2.202867 \\ \mathrm{H} & 1.658342 & -4.541679 & -1.684348 \\ \mathrm{H} & 2.962009 & -4.14346 & -0.54086 \\ \mathrm{H} & -0.000159 & 0.00004 & -0.000103\end{array}$

(e) Filled cage $-\mathrm{H}$ atom exit transition state, UB3LYP/3-21+G*

Total energy $=-3522.26647674$ hartree

$\begin{array}{llll}\mathrm{Si} & 0.000000 & 2.267254 & 1.611575 \\ \mathrm{Si} & 2.267254 & 0.000000 & 1.611575 \\ \mathrm{Si} & -2.267254 & 0.000000 & 1.611575 \\ \mathrm{Si} & 0.000000 & -2.267254 & 1.611575 \\ \mathrm{Si} & 0.000000 & 2.254651 & -1.626363 \\ \mathrm{Si} & 2.254651 & 0.000000 & -1.626363 \\ \mathrm{Si} & -2.254651 & 0.000000 & -1.626363 \\ \mathrm{Si} & 0.000000 & -2.254651 & -1.626363 \\ \mathrm{O} & 0.000000 & 2.576690 & -0.007116 \\ \mathrm{O} & 2.576690 & 0.000000 & -0.007116 \\ \mathrm{O} & -2.576690 & 0.000000 & -0.007116 \\ \mathrm{O} & 0.000000 & -2.576690 & -0.007116 \\ \mathrm{O} & 1.403288 & 1.403288 & 1.930136 \\ \mathrm{O} & -1.403288 & -1.403288 & 1.930136 \\ \mathrm{O} & -1.403288 & 1.403288 & 1.930136 \\ \mathrm{O} & 1.403288 & -1.403288 & 1.930136 \\ \mathrm{O} & 1.334334 & 1.334334 & -1.945964 \\ \mathrm{O} & -1.334334 & 1.334334 & -1.945964 \\ \mathrm{O} & 1.334334 & -1.334334 & -1.945964 \\ \mathrm{O} & -1.334334 & -1.334334 & -1.945964 \\ \mathrm{C} & 0.000000 & 3.814548 & 2.622800 \\ \mathrm{C} & 3.814548 & 0.000000 & 2.622800 \\ \mathrm{C} & -3.814548 & 0.000000 & 2.622800 \\ \mathrm{C} & 0.000000 & -3.814548 & 2.622800 \\ \mathrm{C} & 3.804109 & 0.000000 & -2.634514 \\ \mathrm{C} & -3.804109 & 0.000000 & -2.634514 \\ \mathrm{C} & 0.000000 & 3.804109 & -2.634514 \\ \mathrm{C} & 0.000000 & -3.804109 & -2.634514 \\ \mathrm{H} & -0.890904 & 4.419759 & 2.408314 \\ \mathrm{H} & 0.890904 & 4.419759 & 2.408314 \\ \mathrm{H} & 0.000000 & 3.574104 & 3.694647 \\ \mathrm{H} & 4.419759 & -0.890904 & 2.408314 \\ \mathrm{H} & 4.419759 & 0.890904 & 2.408314 \\ \mathrm{H} & 3.574104 & 0.000000 & 3.694647 \\ \mathrm{H} & -4.419759 & 0.890904 & 2.408314 \\ \mathrm{H} & -4.419759 & -0.890904 & 2.408314 \\ \mathrm{H} & -3.574104 & 0.000000 & 3.694647\end{array}$




$\begin{array}{llll}\mathrm{H} & 0.890904 & -4.419759 & 2.408314 \\ \mathrm{H} & -0.890904 & -4.419759 & 2.408314 \\ \mathrm{H} & 0.000000 & -3.574104 & 3.694647 \\ \mathrm{H} & -0.889971 & 4.410640 & -2.418676 \\ \mathrm{H} & 0.889971 & 4.410640 & -2.418676 \\ \mathrm{H} & 0.000000 & 3.566302 & -3.707015 \\ \mathrm{H} & 4.410640 & -0.889971 & -2.418676 \\ \mathrm{H} & 4.410640 & 0.889971 & -2.418676 \\ \mathrm{H} & 3.566302 & 0.000000 & -3.707015 \\ \mathrm{H} & -4.410640 & 0.889971 & -2.418676 \\ \mathrm{H} & -4.410640 & -0.889971 & -2.418676 \\ \mathrm{H} & -3.566302 & 0.000000 & -3.707015 \\ \mathrm{H} & 0.889971 & -4.410640 & -2.418676 \\ \mathrm{H} & -0.889971 & -4.410640 & -2.418676 \\ \mathrm{H} & 0.000000 & -3.566302 & -3.707015 \\ \mathrm{H} & 0.000000 & 0.000000 & 1.975866\end{array}$

\section{3. $\mathrm{GeSi}_{7} \mathrm{O}_{12} \mathrm{H}_{8}$}

(a) Empty cage, optimized geometry, B3LYP/6-31G*

Total energy $=-5010.09406368$ hartree

$\begin{array}{llll}\mathrm{Ge} & 2.609584 & 0.012312 & -0.001569 \\ \mathrm{H} & 4.118087 & 0.022385 & -0.002082 \\ \mathrm{O} & 2.027819 & -0.137800 & 1.665597 \\ \mathrm{Si} & 0.680500 & -0.224988 & 2.599504 \\ \mathrm{H} & 1.102658 & -0.344891 & 3.997955 \\ \mathrm{O} & -0.231442 & 1.130709 & 2.426413 \\ \mathrm{Si} & -1.15311 & 2.10408 & 1.478983 \\ \mathrm{H} & -1.643789 & 3.232884 & 2.273333 \\ \mathrm{O} & -0.242948 & 2.668137 & 0.234769 \\ \mathrm{Si} & 0.664481 & 2.369656 & -1.102147 \\ \mathrm{H} & 1.080716 & 3.643900 & -1.694929 \\ \mathrm{O} & 2.015707 & 1.525487 & -0.706818 \\ \mathrm{O} & -2.435749 & 1.259224 & 0.893176 \\ \mathrm{Si} & -2.969404 & -0.014268 & 0.001709 \\ \mathrm{H} & -4.434205 & -0.024707 & 0.004331 \\ \mathrm{O} & -2.430816 & 0.126978 & -1.544579 \\ \mathrm{Si} & -1.145449 & 0.220423 & -2.564685 \\ \mathrm{H} & -1.631027 & 0.336851 & -3.941828 \\ \mathrm{O} & -0.238029 & 1.536771 & -2.193381 \\ \mathrm{O} & -0.224268 & -1.131044 & -2.424239 \\ \mathrm{Si} & 0.689549 & -2.134189 & -1.49798 \\ \mathrm{H} & 1.114742 & -3.280304 & -2.30658 \\ \mathrm{O} & 2.036383 & -1.361988 & -0.962805\end{array}$




$\begin{array}{llll}\mathrm{O} & -0.210099 & -2.672017 & -0.232697 \\ \mathrm{Si} & -1.128023 & -2.341391 & 1.087545 \\ \mathrm{H} & -1.609356 & -3.595625 & 1.671571 \\ \mathrm{O} & -0.21875 & -1.538343 & 2.193226 \\ \mathrm{O} & -2.415821 & -1.417988 & 0.652266\end{array}$

(b) Filled cage, optimized geometry, B3LYP/6-31G*

Total energy $=-5010.58346399$ hartree

$\begin{array}{llll}\mathrm{Ge} & -2.602948 & 0.022691 & -0.00011 \\ \mathrm{H} & -4.111169 & 0.031708 & 0.000148 \\ \mathrm{O} & -2.036471 & -0.824122 & -1.453249 \\ \mathrm{Si} & -0.688736 & -1.309024 & -2.259231 \\ \mathrm{H} & -1.11792 & -2.004612 & -3.475932 \\ \mathrm{O} & 0.236548 & -0.01573 & -2.681508 \\ \mathrm{Si} & 1.153176 & 1.27358 & -2.235218 \\ \mathrm{H} & 1.638715 & 1.957817 & -3.436164 \\ \mathrm{O} & 0.243788 & 2.317598 & -1.349141 \\ \mathrm{Si} & -0.658873 & 2.617129 & -0.006329 \\ \mathrm{H} & -1.063893 & 4.025756 & -0.009833 \\ \mathrm{O} & -2.02322 & 1.700246 & -0.005044 \\ \mathrm{O} & 2.442545 & 0.764448 & -1.349288 \\ \mathrm{Si} & 2.965795 & -0.018689 & -0.000244 \\ \mathrm{H} & 4.43047 & -0.045137 & 0.000042 \\ \mathrm{O} & 2.442037 & 0.77252 & 1.343813 \\ \mathrm{Si} & 1.153231 & 1.284569 & 2.228904 \\ \mathrm{H} & 1.639592 & 1.974635 & 3.426177 \\ \mathrm{O} & 0.242374 & 2.323907 & 1.338813 \\ \mathrm{O} & 0.23811 & -0.00333 & 2.682197 \\ \mathrm{Si} & -0.688434 & -1.297719 & 2.265875 \\ \mathrm{H} & -1.117977 & -1.987336 & 3.485846 \\ \mathrm{O} & -2.035747 & -0.815322 & 1.457846 \\ \mathrm{O} & 0.199094 & -2.337204 & 1.349696 \\ \mathrm{Si} & 1.113173 & -2.588497 & 0.00649 \\ \mathrm{H} & 1.601738 & -3.969609 & 0.009892 \\ \mathrm{O} & 0.199671 & -2.343479 & -1.338283 \\ \mathrm{O} & 2.397875 & -1.561817 & 0.004206 \\ \mathrm{H} & 0.131294 & 0.010038 & -0.000596\end{array}$

(c) Filled cage, distal face $\mathrm{H}$ atom exit transition state, UB3LYP/6-31G*

Total energy $=-5010.54390830$ hartree

$\begin{array}{llll}\mathrm{Si} & -0.179661 & 2.226883 & 1.721124 \\ \mathrm{Ge} & 2.114495 & 0.000000 & -1.538189\end{array}$




$\begin{array}{llll}\mathrm{Si} & -0.179661 & -2.226883 & 1.721124 \\ \mathrm{Si} & -2.414399 & 0.000000 & -1.456809 \\ \mathrm{Si} & -2.409001 & 0.000000 & 1.739305 \\ \mathrm{Si} & -0.209403 & -2.245752 & -1.480014 \\ \mathrm{Si} & -0.209403 & 2.245752 & -1.480014 \\ \mathrm{Si} & 2.075221 & 0.000000 & 1.737789 \\ \mathrm{O} & -1.590860 & 1.413022 & 2.083914 \\ \mathrm{O} & -0.193361 & 2.594930 & 0.124105 \\ \mathrm{O} & 1.233962 & 1.406428 & 2.048136 \\ \mathrm{O} & 1.161681 & -1.448657 & -1.905272 \\ \mathrm{O} & 1.161681 & 1.448657 & -1.905272 \\ \mathrm{O} & 2.567361 & 0.000000 & 0.173593 \\ \mathrm{O} & -1.590860 & -1.413022 & 2.083914 \\ \mathrm{O} & -0.193361 & -2.594930 & 0.124105 \\ \mathrm{O} & 1.233962 & -1.406428 & 2.048136 \\ \mathrm{O} & -2.785133 & 0.000000 & 0.142720 \\ \mathrm{O} & -1.538798 & -1.341102 & -1.822026 \\ \mathrm{O} & -1.538798 & 1.341102 & -1.822026 \\ \mathrm{H} & -0.168877 & 3.450514 & 2.525660 \\ \mathrm{H} & 3.361643 & 0.000000 & -2.386224 \\ \mathrm{H} & -0.168877 & -3.450514 & 2.525660 \\ \mathrm{H} & -3.650182 & 0.000000 & -2.243240 \\ \mathrm{H} & -3.630393 & 0.000000 & 2.547197 \\ \mathrm{H} & -0.267675 & -3.492234 & -2.248753 \\ \mathrm{H} & -0.267675 & 3.492234 & -2.248753 \\ \mathrm{H} & 3.254565 & 0.000000 & 2.607020 \\ \mathrm{H} & -0.177879 & 0.000000 & 2.116160\end{array}$

(d) Filled cage, proximal face $\mathrm{H}$ atom exit transition state, UB3LYP/6-31G*

Total energy $=-5010.55672625$ hartree

$\begin{array}{llll}\mathrm{Si} & -2.617567 & 0.737962 & 0.000000 \\ \mathrm{Si} & 1.163099 & 1.323975 & 2.250215 \\ \mathrm{Ge} & 0.165908 & -2.500523 & 0.000000 \\ \mathrm{Si} & 1.163099 & 1.323975 & -2.250215 \\ \mathrm{Si} & -1.134725 & -0.817260 & -2.409651 \\ \mathrm{Si} & 2.610728 & -0.392200 & 0.000000 \\ \mathrm{Si} & -0.371779 & 2.935150 & 0.000000 \\ \mathrm{Si} & -1.134725 & -0.817260 & 2.409651 \\ \mathrm{O} & -2.199394 & -0.123367 & -1.343045 \\ \mathrm{O} & -1.837200 & 2.180259 & 0.000000 \\ \mathrm{O} & -2.199394 & -0.123367 & 1.343045 \\ \mathrm{O} & 2.238571 & 0.492274 & 1.336193 \\ \mathrm{O} & 0.464187 & 2.507104 & 1.346249 \\ \mathrm{O} & 0.002172 & 0.299934 & 2.807948\end{array}$




$\begin{array}{llll}\mathrm{O} & -0.445254 & -2.163631 & -1.736764 \\ \mathrm{O} & 1.802884 & -1.814057 & 0.000000 \\ \mathrm{O} & -0.445254 & -2.163631 & 1.736764 \\ \mathrm{O} & 0.002172 & 0.299934 & -2.807948 \\ \mathrm{O} & 2.238571 & 0.492274 & -1.336193 \\ \mathrm{O} & 0.464187 & 2.507104 & -1.346249 \\ \mathrm{H} & -4.065023 & 0.959714 & 0.000000 \\ \mathrm{H} & 1.849356 & 1.933301 & 3.391858 \\ \mathrm{H} & 0.219313 & -4.006797 & 0.000000 \\ \mathrm{H} & 1.849356 & 1.933301 & -3.391858 \\ \mathrm{H} & -1.858118 & -1.216602 & -3.621262 \\ \mathrm{H} & 4.048982 & -0.675460 & 0.000000 \\ \mathrm{H} & -0.585664 & 4.384097 & 0.000000 \\ \mathrm{H} & -1.858118 & -1.216602 & 3.621262 \\ \mathrm{H} & -1.092933 & -1.325647 & 0.000000\end{array}$

\section{4. $\mathrm{C}_{8} \mathrm{O}_{12} \mathrm{H}_{8}$}

Optimized energy minimum geometry, B3LYP/6-31+G*

Total energy $=-1212.23874865$ hartree

$\begin{array}{llll}\mathrm{C} & 1.968659 & -0.168571 & 0.947767 \\ \mathrm{H} & 2.945653 & -0.252635 & 1.42061 \\ \mathrm{O} & 1.392343 & 0.948995 & 1.567851 \\ \mathrm{C} & 0.467571 & 1.865884 & 1.048717 \\ \mathrm{H} & 0.698899 & 2.791959 & 1.572382 \\ \mathrm{O} & 0.663384 & 2.183876 & -0.303063 \\ \mathrm{C} & 0.01854 & 1.656673 & -1.431672 \\ \mathrm{H} & 0.027208 & 2.480228 & -2.143615 \\ \mathrm{O} & 0.729222 & 0.640626 & -2.085129 \\ \mathrm{C} & 1.520539 & -0.377447 & -1.532415 \\ \mathrm{H} & 2.272114 & -0.564393 & -2.297563 \\ \mathrm{O} & 2.268615 & 0.010575 & -0.41138 \\ \mathrm{O} & -1.345275 & 1.386969 & -1.249216 \\ \mathrm{C} & -1.968658 & 0.168282 & -0.947975 \\ \mathrm{H} & -2.946437 & 0.251242 & -1.419423 \\ \mathrm{O} & -1.391205 & -0.948685 & -1.569117 \\ \mathrm{C} & -0.468113 & -1.865909 & -1.048588 \\ \mathrm{H} & -0.698515 & -2.792502 & -1.57182 \\ \mathrm{O} & 0.867263 & -1.609885 & -1.391041 \\ \mathrm{O} & -0.665902 & -2.182139 & 0.303478 \\ \mathrm{C} & -0.018997 & -1.656876 & 1.431423 \\ \mathrm{H} & -0.027552 & -2.480496 & 2.143311 \\ \mathrm{O} & 1.34422 & -1.387615 & 1.246781 \\ \mathrm{O} & -0.728018 & -0.639957 & 2.086006\end{array}$




$\begin{array}{llll}\mathrm{C} & -1.520223 & 0.376991 & 1.532926 \\ \mathrm{H} & -2.272716 & 0.563616 & 2.297243 \\ \mathrm{O} & -0.867025 & 1.609706 & 1.393242 \\ \mathrm{O} & -2.266943 & -0.011363 & 0.411309\end{array}$

\section{5. $\mathrm{Ge}_{8} \mathrm{O}_{12} \mathrm{H}_{8}$}

Optimized energy minimum geometry, B3LYP/6-31+G* (5d, $T_{h}$ symmetry)

Total energy $=-17506.1074526$ hartree

$\begin{array}{llll}\mathrm{Ge} & -1.667241 & 1.667241 & 1.667241 \\ \mathrm{Ge} & 1.667241 & 1.667241 & 1.667241 \\ \mathrm{Ge} & -1.667241 & -1.667241 & 1.667241 \\ \mathrm{Ge} & -1.667241 & 1.667241 & -1.667241 \\ \mathrm{Ge} & 1.667241 & -1.667241 & 1.667241 \\ \mathrm{Ge} & 1.667241 & 1.667241 & -1.667241 \\ \mathrm{Ge} & -1.667241 & -1.667241 & -1.667241 \\ \mathrm{Ge} & 1.667241 & -1.667241 & -1.667241 \\ \mathrm{H} & -2.551208 & 2.551208 & 2.551208 \\ \mathrm{H} & 2.551208 & 2.551208 & 2.551208 \\ \mathrm{H} & -2.551208 & -2.551208 & 2.551208 \\ \mathrm{H} & -2.551208 & 2.551208 & -2.551208 \\ \mathrm{H} & 2.551208 & -2.551208 & 2.551208 \\ \mathrm{H} & 2.551208 & 2.551208 & -2.551208 \\ \mathrm{H} & -2.551208 & -2.551208 & -2.551208 \\ \mathrm{H} & 2.551208 & -2.551208 & -2.551208 \\ \mathrm{O} & 0.000000 & 1.715908 & 2.287498 \\ \mathrm{O} & 0.000000 & -1.715908 & 2.287498 \\ \mathrm{O} & 0.000000 & 1.715908 & -2.287498 \\ \mathrm{O} & 0.000000 & -1.715908 & -2.287498 \\ \mathrm{O} & -1.715908 & 2.287498 & 0.000000 \\ \mathrm{O} & 1.715908 & 2.287498 & 0.000000 \\ \mathrm{O} & -1.715908 & -2.287498 & 0.000000 \\ \mathrm{O} & 1.715908 & -2.287498 & 0.000000 \\ \mathrm{O} & -2.287498 & 0.000000 & 1.715908 \\ \mathrm{O} & 2.287498 & 0.000000 & 1.715908 \\ \mathrm{O} & -2.287498 & 0.000000 & -1.715908 \\ \mathrm{O} & 2.287498 & 0.000000 & -1.715908\end{array}$

\section{References}

(19) Frisch, M. J.; Trucks, G. W.; Schlegel, H. B.; Scuseria, G. E.; Robb, M. A.; Cheeseman, J. R.; Zakrzewski, V. G.; Montgomery, J. A.; Stratmann, R. E.; Burant, J. C.; 
Dapprich, S.; Millam, J. M.; Daniels, A. D.; Kudin, K. N.; Strain, M. C.; Farkas, O.; Tomasi, J.; Barone, V.; Cossi, M.; Cammi, R.; Mennucci, B.; Pomelli, C.; Adamo, C.; Clifford, S.; Ochterski, J.; Petersson, G. A.; Ayala, P. Y.; Cui, Q.; Morokuma, K.; Malick, D. K.; Rabuck, A. D.; Raghavachari, K.; Foresman, J. B.; Cioslowski, J.; Ortiz, J. V.; Baboul, A. G.; Stefanov, B. B.; Liu, G.; Liashenko, A.; Piskorz, P.; Komaromi, I.; Gomperts, R.; Martin, R. L.; Fox, D. J.; Keith, T.; Al-Laham, M. A.; Peng, C. Y.; Nanayakkara, A.; Gonzalez, C.; Challacombe, M.; Gill, P. M. W.; Johnson, B. G.; Chen, W.; Wong, M. W.; Andres, J. L.; Head-Gordon, M.; Replogle, E. S.; Pople, J. A.; A.7 ed.; Gaussian, Inc: Pittsburgh PA, 1998. 